\title{
Optimal allocation of power quality monitors based on an improved
}

\section{adaptive genetic algorithm}

\author{
Wang Hong ${ }^{1,2, a}{ }^{*}$, Li Dan ${ }^{3, b}$, Huang Wenqing ${ }^{2, c}$, Dai Yuxing ${ }^{4,2, d}$ \\ ${ }^{1}$ College of Engineering and Design, Hunan Normal University, Changsha, China 410081 \\ ${ }^{2}$ College of Electrical and Information Engineering, Hunan University, Changsha, China 410082 \\ ${ }^{3}$ Hunan Vocational College of Science and Technology, Changsha, China 410004 \\ ${ }^{4}$ College of Physics and Electronic Information Engineering, Wenzhou University, Wenzhou, \\ China 325035 \\ awhdy63@hotmail.com, ${ }^{b}$ lidan861@gmail.com, cihuangwenqing@gmail.com, ddaiyx@hnu.cn
}

\begin{abstract}
Keywords: voltage sag; observability; adaptive genetic algorithm; redundancy; monitor optimization allocation
\end{abstract}

\begin{abstract}
To solve power quality problems caused by voltage sag, an improved adaptive genetic algorithm is presented for the optimal allocation of power quality monitors. The basic idea of the algorithm is that the entire system can be observed when voltage sag occurs on the buses and along the lines, and thus, the minimum placement number of monitors is ensured. The fitness function of the genetic algorithm is adopted to evaluate individuals that randomly generate the placement number and the locations of monitors. The unobserved individuals are changed by applying the improved adaptive crossover operator and the mutation operator. The optimal solution is extracted from multiple eligible solutions by evaluating redundancy. The feasibility of the algorithm is verified on the IEEE30 bus system.
\end{abstract}

\section{Introduction}

With the development of the power industry and the technological updating of electrical equipment, the wide application of all types of sensitive equipment has resulted in numerous power quality problems, which have attracted the attention of the electric power department and users [1]. Among these problems, those caused by voltage sag are more serious and frequent, and thus, have caused enormous economic losses to users [2]. To ensure power quality and electric safety, the electric power department should implement real-time power quality monitoring. The ideal program is to place power quality monitors (PQMs) at each node of a power system to record each possible power quality occurrence in the system. Given limitations in economic cost and technical capability, placing PQMs at each node is impossible at present. Hence, research on the optimal allocation of monitors has practical significance [3].

Scholars both at home and abroad have conducted in-depth research on the optimal allocation method of monitors. Literature [4-6] adopted the integer linear programming method to determine the optimal allocation scheme. Literature [7, 8] derived optimal solutions based on the topology of the power grid. Literature [9] regarded the economic cost of the network and the minimum number of monitors as objective functions and then obtained the solution for the multi-objective optimization problem using a rapid non-dominated sorting genetic algorithm (GA) and the optimal solution using a normalization method. These works exhibited certain limitations 
by failing to consider the influence of voltage sag and other power quality problems on the monitor reach area (MRA). Literature [10] applied binary coding and real coding in the optimal allocation of monitors when the population of the GA is generated, considered fuzzy set theory as the fitness evaluation function, and used MATLAB simulation calculation to obtain the optimal solution. Literature [11] proposed two monitor optimal allocation methods based on fault location and voltage sag. The first method improved fault location to accurately estimate voltage sag on the bus that could not be monitored. This method is applicable to situations without economic cost limitation. The other method combined any two monitors: one was used for fault location and the other was used to detect voltage sag. This method does not need a trigger voltage threshold and requires less number of monitors than the first method.

Using the aforementioned literature as references, the present study considers voltage sag caused by different types of short-circuit faults and the influence of faults occurring on buses and along the lines on the MRA of voltage sag. The minimum number of monitors placed is considered the objective, and the constraint condition of observation of all the monitors on a network is satisfied. In addition, this study establishes the optimal allocation model for the monitors according to a GA, overcomes the drawback of GA prematurity with an improved adaptive crossover operation and mutation operation, and adopts the elitism preserving strategy. The proposed algorithm provides a simple calculation method and maintains the diversity of the optimal solution.

\section{Definition of MRA of voltage sag}

Calculating voltage amplitude when voltage sag occurs at the node. In this study, the amplitude of voltage sag at a short-circuit fault is calculated using a node impedance matrix [12]. Given network parameters and system structure, the node impedance matrix $Z$ can be obtained according to power system analysis [13]. To calculate a short-circuit fault easily, the nominal value of voltage at the nodes of a system before the fault occurs is assumed to be 1.0 p.u. and the metallic earth fault impedance $z_{f}$ is $0 \Omega$. In addition, the transient state process of voltage sag is ignored, and voltage is considered to drop to the minimum value when the fault occurs.

A three-phase short-circuit fault is a symmetrical fault. That is, the lines are symmetrical when a fault occurs; hence, any line can be selected to calculate voltage at different nodes of the network at the fault. The three-phase short-circuit fault is assumed to occur at Node $i$. Thus, the voltage of Node $j[13]$ is

$$
\boldsymbol{V}_{i j}=1-\frac{\boldsymbol{Z}_{i j}}{\boldsymbol{Z}_{i i}} \text {. }
$$

where $V_{i j}$ is the voltage at Node $j$ after the fault of Node $i, Z_{i j}$ is the mutual impedance from Node $i$ to Node $j$, and $Z_{i i}$ is the self-impedance of Node $i$.

Single-phase and two-phase earth faults, as well as two-phase short-circuit fault, are asymmetrical faults. When an asymmetrical fault occurs, the three-phase lines of a system will no longer be symmetrical; hence, the models for the positive, negative, and zero sequence components of a system are developed using the symmetrical component method [13]. According to the asymmetrical fault calculation for the power system, the three-phase voltages at different nodes [14] when an asymmetrical fault occurs are as follows:

Single-phase earth fault 


$$
\left\{\begin{array}{l}
\boldsymbol{V}_{i j a}=1-\frac{\boldsymbol{Z}_{i j}^{(1)}+\boldsymbol{Z}_{i j}^{(2)}+\boldsymbol{Z}_{i j}^{(0)}}{\boldsymbol{Z}_{i i}^{(1)}+\boldsymbol{Z}_{i i}^{(2)}+\boldsymbol{Z}_{i i}^{(0)}} \\
\boldsymbol{V}_{i j b}=\alpha^{2}-\frac{a^{2} \boldsymbol{Z}_{i j}^{(1)}+\alpha \boldsymbol{Z}_{i j}^{(2)}+\boldsymbol{Z}_{i j}^{(0)}}{\boldsymbol{Z}_{i i}^{(1)}+\boldsymbol{Z}_{i i}^{(2)}+\boldsymbol{Z}_{i i}^{(0)}} \\
\boldsymbol{V}_{i j c}=\alpha-\frac{\alpha \boldsymbol{Z}_{i j}^{(1)}+\alpha^{2} \boldsymbol{Z}_{i j}^{(2)}+\boldsymbol{Z}_{i j}^{(0)}}{\boldsymbol{Z}_{i i}^{(1)}+\boldsymbol{Z}_{i i}^{(2)}+\boldsymbol{Z}_{i i}^{(0)}}
\end{array} .\right.
$$

Two-phase short-circuit fault

$$
\left\{\begin{array}{l}
\boldsymbol{V}_{i j a}=1-\frac{\boldsymbol{Z}_{i j}^{(1)}-\boldsymbol{Z}_{i j}^{(2)}}{\boldsymbol{Z}_{i i}^{(1)}+\boldsymbol{Z}_{i i}^{(2)}} \\
\boldsymbol{V}_{i j b}=\alpha^{2}-\frac{\alpha^{2} \boldsymbol{Z}_{i j}^{(1)}-\alpha \boldsymbol{Z}_{i j}^{(2)}}{\boldsymbol{Z}_{i i}^{(1)}+\boldsymbol{Z}_{i i}^{(2)}} \\
\boldsymbol{V}_{i j c}=\alpha-\frac{\alpha \boldsymbol{Z}_{i j}^{(1)}-\alpha^{2} \boldsymbol{Z}_{i j}^{(2)}}{\boldsymbol{Z}_{i i}^{(1)}+\boldsymbol{Z}_{i i}^{(2)}} .
\end{array} .\right.
$$

Two-phase earth fault

$$
\left\{\begin{array}{l}
\boldsymbol{V}_{i j a}=1-\frac{\boldsymbol{Z}_{i j}^{(1)}\left(\boldsymbol{Z}_{i i}^{(2)}+\boldsymbol{Z}_{i i}^{(0)}\right)-\boldsymbol{Z}_{i j}^{(2)} \boldsymbol{Z}_{i i}^{(0)}-\boldsymbol{Z}_{i j}^{(0)} \boldsymbol{Z}_{i i}^{(2)}}{\boldsymbol{Z}_{i i}^{(1)} \boldsymbol{Z}_{i i}^{(2)}+\boldsymbol{Z}_{i i}^{(2)} \boldsymbol{Z}_{i i}^{(0)}+\boldsymbol{Z}_{i i}^{(0)} \boldsymbol{Z}_{i i}^{(1)}} \\
\boldsymbol{V}_{i j b}=\alpha^{2}-\frac{\alpha^{2} \boldsymbol{Z}_{i j}^{(1)}\left(\boldsymbol{Z}_{i i}^{(2)}+\boldsymbol{Z}_{i i}^{(0)}\right)-\alpha \boldsymbol{Z}_{i j}^{(2)} \boldsymbol{Z}_{i i}^{(0)}-\boldsymbol{Z}_{i j}^{(0)} \boldsymbol{Z}_{i i}^{(2)}}{\boldsymbol{Z}_{i i}^{(1)} \boldsymbol{Z}_{i i}^{(2)}+\boldsymbol{Z}_{i i}^{(2)} \boldsymbol{Z}_{i i}^{(0)}+\boldsymbol{Z}_{i i}^{(0)} \boldsymbol{Z}_{i i}^{(1)}} \\
\boldsymbol{V}_{i j c}=\alpha-\frac{\alpha \boldsymbol{Z}_{i j}^{(1)}\left(\boldsymbol{Z}_{i i}^{(2)}+\boldsymbol{Z}_{i i}^{(0)}\right)-\alpha^{2} \boldsymbol{Z}_{i j}^{(2)} \boldsymbol{Z}_{i i}^{(0)}-\boldsymbol{Z}_{i j}^{(0)} \boldsymbol{Z}_{i i}^{(2)}}{\boldsymbol{Z}_{i i}^{(1)} \boldsymbol{Z}_{i i}^{(2)}+\boldsymbol{Z}_{i i}^{(2)} \boldsymbol{Z}_{i i}^{(0)}+\boldsymbol{Z}_{i i}^{(0)} \boldsymbol{Z}_{i i}^{(1)}}
\end{array} .\right.
$$

$\boldsymbol{V}_{i j a}, \boldsymbol{V}_{i j b}$, and $\boldsymbol{V}_{i j c}$ in Equations (2) to (4) are the three-phase voltages (a, b, and c) at Node $j$ when Node $i$ fails. The superscripts (1), (2), and (0) represent positive, negative, and zero sequences, respectively.

Calculating voltage amplitude when voltage sag occurs on the lines. When a fault occurs on the lines and causes voltage sag, the fault point can be regarded as an independent node. Thus, the number of nodes in the system is more than that in the previous system at this time.

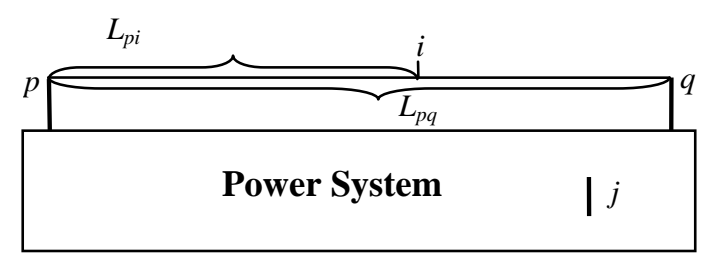

Fig. 1 Diagram of a Power System

Fig.1 presents the diagram of a power system. Node $i$ is assumed as the fault point on transmission line $L_{p q}$, which connects Node $p$ and Node $q$. The location of Fault Point $i$ can be represented by Parameter $\omega$ [value range $(0,1)$ ]. That is, $i$ moves on $L_{p q}$. Thus, $\omega$ is defined as follows:

$$
\omega=\frac{L_{p i}}{L_{p q}} \quad 0 \leq \omega \leq 1
$$

where $L_{p i}$ represents the distance between Node $p$ and Fault Point $i$. When $\omega=0$ or 1 , a fault occurs at Node $p$ or Node $q$.

Equations (1) to (4) should calculate voltage according to $Z_{i i}$ and $Z_{i j}$. When a fault occurs on the line, Fault Point $i$ is not fixed on $L_{p q}$; hence, calculating $Z_{i i}$ and $Z_{i j}$ directly is impossible. According to Equation (5) and Reference [15], the calculation equations of $Z_{i i}$ and $Z_{i j}$ are as 
follows:

$$
\begin{aligned}
& \boldsymbol{Z}_{i i}^{(m)}=\omega^{2}\left(\boldsymbol{Z}_{p p}^{(m)}+\boldsymbol{Z}_{q q}^{(m)}-2 \boldsymbol{Z}_{p q}^{(m)}-z_{p q}^{(m)}\right)-\omega\left(2 \boldsymbol{Z}_{p p}^{(m)}-2 \boldsymbol{Z}_{p q}^{(m)}-z_{p q}^{(m)}\right)+\boldsymbol{Z}_{p p}^{(m)} . \\
& \boldsymbol{Z}_{i j}^{(m)}=\omega\left(\boldsymbol{Z}_{q j}^{(m)}-\boldsymbol{Z}_{p j}^{(m)}\right)+\boldsymbol{Z}_{p j}^{(m)} .
\end{aligned}
$$

In Equations (6) and (7), $Z_{p p}$ and $Z_{q q}$ denote the self-impedances of Node $p$ and Node $q$, respectively, in the node impedance matrix; $Z_{p q}$ is the mutual impedance between $p$ and $q$; and $z_{p q}$ is the impedance of $z_{p q} . m=1,2,0$ represent positive, negative, and zero sequences, respectively.

The voltage values at different phases of the nodes when voltage sag occurs on the lines of the power system can be obtained by substituting Equations (6) and (7) into Equations (1) to (4).

Definition of MRA.MRA refers to the network scope that a given monitor position can reach[15]. That is, if a fault occurs in the MRA, then the monitor will be triggered to capture the voltage sag event. The observability of fault points in entire whole network is closely related to MRA. A reasonable monitor point location can protect the observability of a power system.

In this design, an MRM with $n$ rows and $k$ columns is used to represent MRA. The voltage values at different nodes obtained in Section 1.1 are compared with a set of threshold values $v_{t}$ (typically 0.9 p.u.). The results are used to represent the situations of voltage sag. When voltage at a node is greater than the set threshold $v_{t}$, the corresponding element value in the $M R M$ is 0 , which indicates that no voltage sag occurs; otherwise, the value is 1 , which indicates that a voltage sag occurs. The process that constitutes the MRM is as follows:

$$
\boldsymbol{M R M}_{i j}=\left\{\begin{array}{ll}
1, & \boldsymbol{V}_{i j} \leq v_{t} \\
0, & \boldsymbol{V}_{i j}>v_{t}
\end{array} .\right.
$$

In Equation (8), the value range of $i$ in the $M R M$ is $[1, n]$, which represents the number of fault points in the power system; the value range of $j$ is $[1, k]$, which represents the number of nodes in the power system.

Under an asymmetrical short-circuit fault, the minimum value among $V_{i j a}, V_{i j b}$, and $V_{i j c}$ is selected to be compared with the threshold $v_{t}$ to obtain the MRM under the asymmetrical short-circuit fault as follows:

$$
\boldsymbol{M R M}_{i j}=\left\{\begin{array}{ll}
1, & \min \left(v_{i j a}, v_{i j b}, v_{i j c}\right) \leq v_{t} \\
0, & \min \left(v_{i j a}, v_{i j b}, v_{i j c}\right)>v_{t}
\end{array} .\right.
$$

\section{PQM optimal allocation method based on an improved adaptive GA (IAGA)}

IAGA. A simple GA (SGA) consists of population initialization, fitness evaluation, selection operation, crossover operation, and mutation operation. This algorithm exhibits the disadvantages of slow convergence speed and ease of falling into a local optimal solution [16]. Thus, adaptive GA (AGA) is proposed, which provides automatically adjusted crossover and mutation probabilities along with the change in individual fitness value [17]. This algorithm increases convergence precision and speed to a certain extent and provides diversified solutions. However, considering the minimal or lack of difference between the maximum fitness value of a population and the superior individuals at the beginning of the evolution, crossover and mutation operations cannot act on superior individuals. Consequently, the algorithm develops toward the possibility of 
a local optimal solution. In the present study, the IAGA proposed in Literature [17] is adopted for the optimal allocation of PQMs. This algorithm automatically adjusts crossover and mutation probabilities with the evolutional attenuation factor and the individual fitness value along with time. IAGA exhibits the advantages of easy realization and high computational efficiency. It can also prevent algorithm prematurity and can obtain a global optimal solution.

PQM optimal allocation method of IAGA. Population .Population refers to a group of innumerable randomly generated individuals, namely, chromosomes. The GA is applied in the optimal allocation of PQMs. The binary coded vectors are adopted to represent individuals. Thus, the binary allocation (BA) vector is as follows:

$$
\boldsymbol{B} A(i)= \begin{cases}1, & \text { The installation of monitoring points on the node } \mathrm{i} \\ 0, & \text { No installation of monitoring points on the node } \mathrm{i}\end{cases}
$$

where $B A(i)$ denotes the elements of $B A$, namely, the genes of individuals. Table 1 shows the $B A$ example of a power system with nine buses. The monitors are set at Buses 1,3 , and 8 . If a monitor needs to be installed at a node, then the corresponding binary component elements to the node can be placed in Bus 1.

Table $1 B A$ Example of a Power System with Nine Buses

\begin{tabular}{|l|l|l|l|l|l|l|l|l|}
\hline 1 & $(2)$ & $(3)$ & $(4)$ & $(5)$ & $(6)$ & $(7)$ & $(8)$ & $(9)$ \\
\hline 1 & 0 & 1 & 0 & 0 & 0 & 0 & 1 & 0 \\
\hline
\end{tabular}

According to the definition of $B A$, the objective function of the monitor optimization problem is as follows:

$$
f_{o b j}=\min \sum_{i=1}^{N} \boldsymbol{B} \boldsymbol{A}(i)
$$

where $N$ represents the number of buses. This function indicates that the number of PQMs is the minimum.

Fitness evaluation. The evaluation of the advantages and disadvantages of each individual is realized through fitness evaluation. In terms of PQM optimal allocation, the fitness function should minimize the installation number of PQMs and should select the best installation locations to allow monitoring of the entire power system.

Redundant vector $R$ is obtained by multiplying the known $M R M$ by the transpose of $B A$. The equation is as follows:

$$
\boldsymbol{R}=\boldsymbol{M R} \boldsymbol{M} * \boldsymbol{B} \boldsymbol{A}^{t} .
$$

where $R$ is a column vector with $n$ rows, $n$ is the number of fault points, and the $R(j)$ of each element in $R$ represents the monitoring times of the fault point. When $R(j)=0$, the jth fault point cannot be monitored. When each element in $\mathrm{R}$ is greater than or equal to 1 , all the fault points in the system can be reached by the monitors.

According to the situation in which the individual fitness of the GA must be greater than 0 and should satisfy the conditions of the minimum objective function, and the monitor should reach all the fault points in the system, the fitness function can be defined as follows: 


$$
\max F=C_{\max }-\sum_{i=1}^{N} \boldsymbol{B A}(i)-c * U
$$

where $C_{\max }$ is a positive number that can ensure that fitness function $F$ is greater than $0 ; U$ is the number of elements with $R(j)=0$ in redundant vector $R$, namely, the number of fault points that the monitors cannot reach; and $c$ is the penalty coefficient of $U(U>0)$. This coefficient can be adjusted according to the size of the power system.

Selection operation. Selection operation is the process of calculating the fitness of each individual according to the fitness function, and then selecting superior individuals and eliminating weak ones based on the calculation. Several selection strategies can be applied in the GA. The tournament selection method is adopted in this study. The tournament size $C$ is two. Any two individuals in the population are randomly combined. Their fitness values are then compared. The individuals with large fitness values are directly copied to the parent-offspring population in the next cycle. This method can ensure the high survival rate of individuals with good fitness. In addition, this method does not need to calculate the selection probability required by the roulette selection operation. In doing so, the execution efficiency of the algorithm is improved.

Adaptive crossover and mutation operations. The crossover and mutation operations in GA are used to generate diversified solutions. These operations cooperate with each other to complete the global and local search of the solution space. Based on the crossover probability $P_{c}$ and the mutation probability $P_{m}$ in IAGA proposed in Literature [17], the computation equations adopted in this study are as follows:

$$
\begin{gathered}
P_{c}=\left\{\begin{array}{l}
F(t) *\left[k_{0}+\left(k_{1}+k_{0}\right) \frac{f_{\text {max }}-f^{\prime}}{f_{\text {max }}-f_{\text {avg }}}\right], \quad f^{\prime} \geq f_{\text {avg }} \\
k_{1}, \quad f^{\prime}<f_{\text {avg }}
\end{array}\right. \\
P_{m}=\left\{\begin{array}{ll}
F(t) *\left[k_{2}+\left(k_{3}-k_{2}\right) \frac{f_{\text {max }}-f}{f_{\text {max }}-f_{\text {avg }}}\right], & f \geq f_{\text {avg }} \\
k_{3}, & f<\text { favg }
\end{array} .\right.
\end{gathered}
$$

In Equations (14) and (15), $F(t)=\lambda e^{-\beta(t / T)^{\varphi}}$ is the evolutional attenuation factor; $t$ is the evolution time; $T$ is the total evolutional generation; and $\lambda=1, \varphi=5, \beta=30, k_{0}=k_{2}=$ $0.5, k_{1}=k_{3}=1 . f_{\max }$ and $f_{\text {avg }}$ are the maximum and average fitness values of the population, respectively; $f^{\prime}$ is the large fitness value in two individuals that require crossover; and $f$ is the fitness value of individuals that require mutation.

Crossover operation is mainly performed to combine new individuals. Crossover modes have many types. In this study, single-point crossover is adopted according to the crossover probability in Equation (14). This type is simple to realize and does not damage the original solution at a large scale. When the fitness values of new individuals generated by the crossover operation no longer evolve and fail to reach the optimal, the algorithm prematurely converges. The alternative mutation is performed on binary coded individuals according to the mutation probability in Equation (15), that is, 0 becomes 1 and 1 becomes 0 . Therefore, new individuals are produced.

To ensure that the crossover and mutation operations do not damage the optimal solution, this study adopts the elitism individual preservation strategy [18] to regard individuals with large 
fitness values directly as the parent-offspring population in the next generation. Thus, crossover and mutation operations are avoided.

Termination condition. The operations in Sections 2.2.2 to 2.2.4 are repeated. When the fitness values of the individuals in the population reach 20 times and no longer change in the iteration process of IAGA, the allocation scheme in which the number of monitors is the minimum and the entire network can be reached by the monitors is adopted. Then, the operation can exit the circle.

Optimal solution evaluation. Several solutions that satisfy the conditions can be obtained by optimizing via IAGA. In practice, the decision maker only needs to select an optimal solution as the final implementation plan. Among the solutions in which the objective function $f_{o b j}$ is the minimum and the entire power system can be reached by the monitors, the optimal solution can be obtained by evaluating redundancy $r$, which is defined as follows:

$$
r=\sum_{i=1}^{M} \boldsymbol{R}(i) / M
$$

where $R(i)$ is the value of each element of redundant vector $R$ in Equation (11), and $M$ is the number of fault points. The greater $r$ is, the more times the fault points are monitored in the system, on average. The individual with the largest $r$ when the objective function is the minimum is selected as the optimal solution. The value range of $r$ is $\left[1, f_{o b j}\right]$, which indicates that each fault point is monitored once at least and $f_{o b j}$ times at most.

\section{Example simulation and analysis}

The IEEE30 bus system is shown in Fig. 2. This system consists of 6 generators, 30 buses, 37 lines, and 4 transformers. The data are obtained from Literature [19].

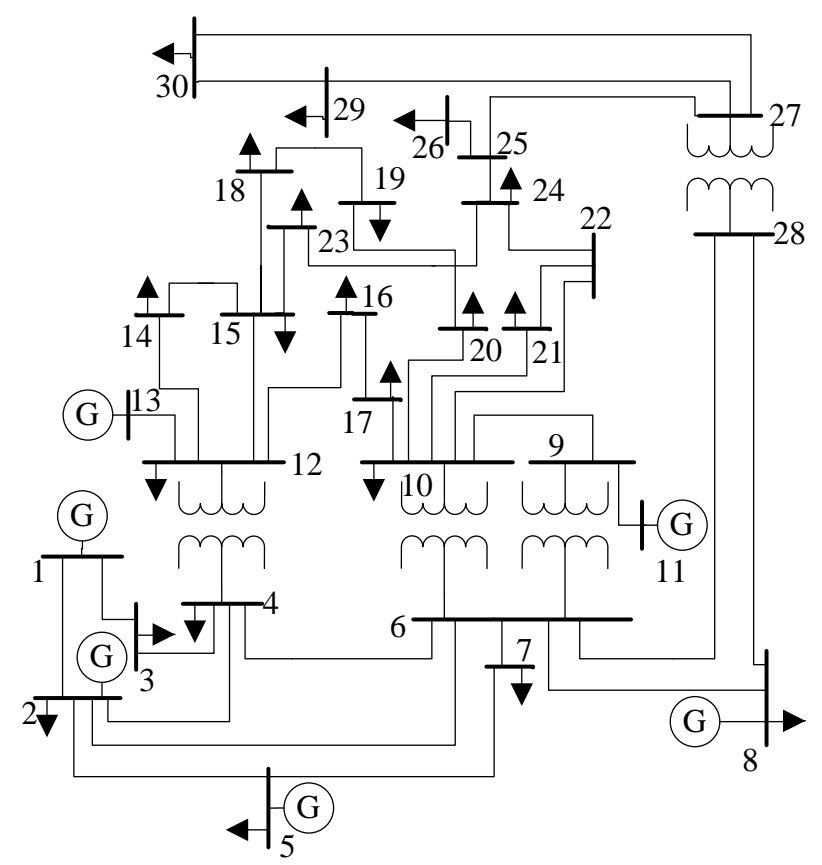

Fig. 2 Single Line Diagram of the IEEE30 Bus System

The proposed model is verified using the IEEE30 bus system. PQM optimal allocation is simulated from two aspects: (1) the fault occurs on the bus and (2) the fault occurs on the bus and the lines. The AGA program and the optimal allocation of the monitors are written using MATLAB 2007 to obtain the simulation calculation results. 
Table 2 shows the initial experimental data of the IAGA optimal model for the IEEE30 bus system.

Table 2 Initial Experimental Data of the IAGA Optimal Model for the IEEE30 Bus System

\begin{tabular}{lcc}
\hline \multicolumn{1}{c}{ Location of Fault Point } & Bus & Bus and Line \\
\hline BA Length & 30 & 32 \\
Penalty Factor $c$ in Fitness Function $F$ & 4 & 4 \\
$C_{\max }$ in Fitness Function $F$ & 120 & 130 \\
Population Size & 50 & 50 \\
Maximum Number of Iterations & 100 & 100 \\
\hline
\end{tabular}

Simulation and analysis of fault points on the buses. Considering that the IEEE30 bus system does not identify which buses have fault points, this study performs simulation calculation when faults occur on the buses. Various types of faults result in different monitor observable matrices. Thus, the optimal allocation schemes of the monitors also vary.

Table 3 Optimal Allocation Schemes of the Monitors under Different Types of Faults When $v_{t}=$ 0.9 p.u.

\begin{tabular}{ccccc}
\hline Fault Type & $\begin{array}{c}\text { Number of Monitors } \\
f_{o b j}\end{array}$ & $\begin{array}{c}\text { Redundancy } \\
\text { Focation of }\end{array}$ & $\begin{array}{c}f_{o b j} / r \\
\text { Bus }\end{array}$ & \\
\hline Three-phase Short-circuit Fault & 1 & 1 & 4 & 1 \\
\hline Single-phase Earth Fault & 2 & 1.61 & 425 & 0.805 \\
\hline Two-phase Short-circuit Fault & 1 & 1 & 24 & 1 \\
\hline Two-phase Earth Fault & 1 & 1 & 24 & 1 \\
\hline Any Fault Type & 2 & 1.61 & 425 & 0.805 \\
\hline
\end{tabular}

Table 3 shows the optimal allocation schemes of the monitors under different types of faults when $v_{t}=0.9$ p.u. According to Table 3, two-phase and three-phase short-circuit faults, as well as two-phase earth fault, require only one monitor to complete observation of the entire system. However, the monitors under different types of faults are placed in various positions. Single-phase earth fault requires two monitors, which should be allocated to Buses 4 and 25, to observe the entire network. This number is one more than those required by the other three types of short-circuit fault. This finding indicates that a single-phase earth fault occurs more frequently and causes significant losses. The allocation scheme for any fault type is the same as that for a single-phase short-circuit fault.

Table 4 Optimal Allocation Schemes of the Monitors under Different Types of Faults When $v_{t}=$ 0.8 p.u.

\begin{tabular}{ccccc}
\hline Fault Type & Number of Monitors Redundancy Location of & \multirow{2}{*}{$f_{\text {obj }} / r$} \\
& $f_{o b j}$ & $r$ & Bus & \\
\hline Three-phase Short-circuit Fault & 3 & 1.91 & 71427 & 0.637 \\
Single-phase Earth Fault & 3 & 2.12 & 41429 & 0.707 \\
Two-phase Short-circuit Fault & 3 & 2.27 & 31127 & 0.757 \\
Two-phase Earth Fault & 2 & 1.45 & 724 & 0.725 \\
\hline Any Fault Type & 4 & 2.63 & 471429 & 0.658 \\
\hline
\end{tabular}

Table 4 shows the optimal allocation scheme of the monitors under different types of faults when $v_{t}=0.8$ p.u. According to Table 4, two-phase and three-phase short-circuit faults, as well as single-phase earth fault, require three monitors, whereas two-phase earth fault requires only two monitors. Any fault type can approximately adopt the single-phase earth fault allocation scheme. 
However, placing a monitor on Bus 7 is necessary to ensure that different types of faults will be observed. The numbers of monitors required by different types of short-circuit faults shown in Table 4 are higher than those shown in Table 3. Thus, the lower the threshold of the voltage sag, the smaller the MRA and the higher the number of monitors required.

The bus locations listed in Tables 3 and 4 are not unique. Several solutions that satisfy system observability and the minimum number of monitors can be obtained according to IAGA. As discussed in Section 2.3, the allocation scheme is selected by evaluating the size of redundancy. The bus locations in Tables 3 and 4 are all schemes with the maximum redundancy $r$ in several solutions when objective function $f_{o b j}$ is the minimum. In Table 3, except for single-phase earth fault that requires 2 monitors and any fault type with a redundancy of 1.61, the redundancies of the other three short-circuit faults are all 1. In Table 4, the redundancies are all greater than or equal to 1.45. As shown in Tables 3 and 4, redundancy is related to the number of monitors. The higher the number of monitors, the higher the redundancy and the more times the fault points are monitored on average. The ratios of $r$ to $f_{o b j}$ in Table 3 are greater than those in Table 4. Thus, the MRA is reduced with the decline of the voltage sag threshold.

Table 5 IAGA Operation Results under Different Types of Faults When $v_{t}=0.8$ p.u.

\begin{tabular}{cccc}
\hline Fault Type & $\begin{array}{c}\text { Number of Monitors } \\
f_{o b j}\end{array}$ & $\begin{array}{c}\text { Number of } \\
\text { Iterations }\end{array}$ & $\begin{array}{c}\text { Number of } \\
\text { Solutions }\end{array}$ \\
\hline Three-phase Short-circuit Fault & 3 & 26 & 18 \\
Single-phase Earth Fault & 3 & 35 & 32 \\
Two-phase Short-circuit Fault & 3 & 30 & 23 \\
Two-phase Earth Fault & 2 & 19 & 28 \\
\hline
\end{tabular}

Table 5 shows the IAGA operation results under different types of faults when $v_{t}=0.8$ p.u. The number of solutions refers to the number of allocation schemes with the minimum number of monitors. For example, when three-phase short-circuit fault occurs, 18 allocation schemes satisfy the conditions that the number of monitors is 3 and that the entire network is observable. Considering limitations in space, other cases are excluded in this paper. The number of iterations is the time that the algorithm is executed when IAGA converges to the optimal solution. For example, when a three-phase short-circuit fault occurs, IAGA should iterate 26 times to converge to the optimal solution using 3 monitors.

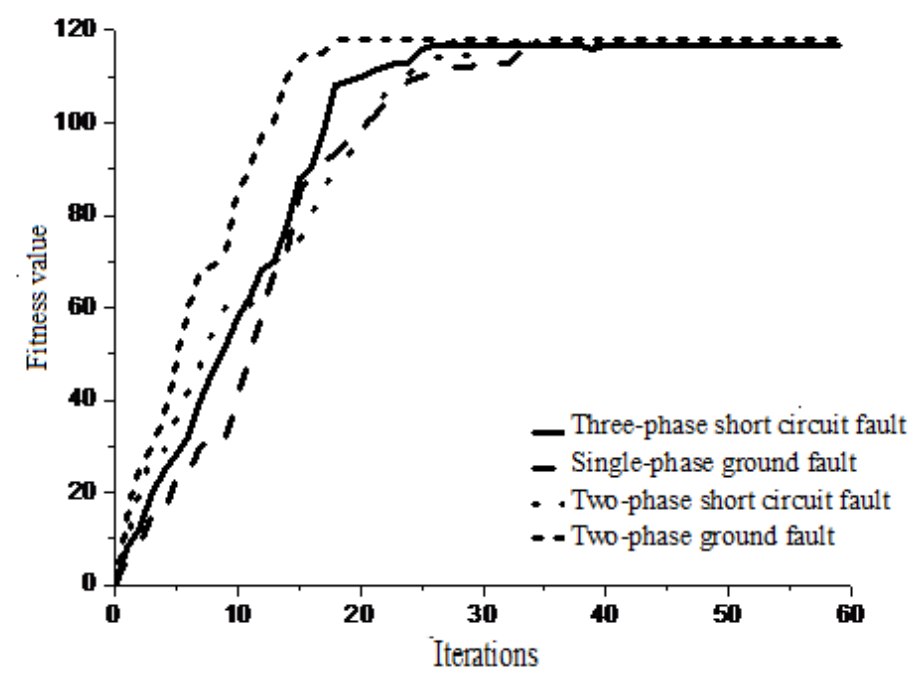

Fig. 3 Relationship Curve between the Number of Iterations and the Fitness Value of IAGA 
Fig. 3 shows the relationship curve between the number of iterations of IAGA and the fitness value, namely, the convergence speed of IAGA. As shown in this figure, the upward trend of the fitness value of IAGA under two-phase earth fault is faster than those under the other three faults. This finding indicates that the convergence speed of this algorithm is fast. The change trends of the fitness value curves of IAGA under the other three fault cases have minimal differences.

Table 6 shows the performance comparison of three algorithms, namely, SGA in Literature [16], AGA in Literature [17], and IAGA in the present study. These algorithms are all the results of the three-phase short-circuit fault in Table 5, in which BA length, population size, and maximum number of iterations are obtained from Table 2. Each algorithm operates 15 times.

Table 6 Performance Comparison of SGA, AGA, and IAGA

\begin{tabular}{cccc}
\hline Algorithm & SGA & AGA & IAGA \\
\hline Optimal Times of Local Convergence & 9 & 6 & 2 \\
Optimal Times of Global Convergence & 1 & 5 & 12 \\
Number of Optimal Solutions & 1 & 7 & 18 \\
\hline
\end{tabular}

As shown in Table 6, the local convergence number of SGA is 9, which is obviously more than those of the other two algorithms. This result indicates that this algorithm can easily fall into local convergence and has difficulty obtaining a global optimal solution. The maximum number of global convergence of IAGA is 12, which obtains 18 optimal solutions. This value is 1 and 7 more than those of SGA and AGA, respectively. This finding reveals that the performance of IAGA is better than those of the other two algorithms. The optimal number of local convergence of AGA is 6, which is 3 less than that of SGA and 4 more than that of IAGA. In addition, both the optimal number of global convergence and the number of optimal solutions of AGA are more than those of SGA and less than those of IAGA. This observation shows that the performance of AGA is better than that of SGA but worse than that of IAGA.

Simulation and analysis of the fault points on the buses and lines. When faults occur on the lines, the calculation method for the amplitude of voltage sag and the MRM are different from those used when faults occur only on the buses. In this study, the faults are assumed to occur on all the buses and two lines, as shown in Table 7. The IAGA optimal model is adopted to solve this problem. Table 2 shows the initial parameters of IAGA.

Table 7 Fault Locations on Two Lines

\begin{tabular}{cc}
\hline Lines between Buses & Fault Locations on the Lines \\
\hline $3-4$ & 0.48 \\
$22-24$ & 0.33 \\
\hline
\end{tabular}

Table 8 Optimization Results of Different Types of Faults Occurring on Buses and Lines When $v_{t}$ $=0.8$

\begin{tabular}{cccl}
\hline Fault Type & $\begin{array}{c}\text { Number of Monitors } \\
\end{array}$ & $\begin{array}{c}\text { Redundancy } \\
f_{\text {obj }}\end{array}$ & Allocation Scheme \\
\hline Three-phase Short-circuit Fault & 3 & 2.09 & 42227 \\
Single-phase Earth Fault & 4 & 2.44 & 452326 \\
Two-phase Short-circuit Fault & 3 & 2.16 & 4524 \\
Two-phase Earth Fault & 3 & 2.18 & 51227 \\
\hline Any Fault Type & 5 & 3.25 & 45222326 \\
\hline
\end{tabular}

Table 8 shows the optimization results of different types of faults occurring on the buses and lines when $v_{t}=0.8$. As shown in the table, any fault type requires five monitors, which is more 
than the other four short-circuit faults. Single-phase earth fault requires four monitors, whereas the other three short-circuit faults require only three monitors to observe the entire network. The comparison of the number of monitors in Table 8 and the results in Table 4 show that the minimum numbers of monitors required by single-phase and two-phase earth faults, as well any fault type in Table 8, are one more than those indicated in Table 4; the numbers of monitors of two-phase and three-phase short-circuit faults remain three. Thus, in case the faults occur on the same bus locations, adding the fault points on the lines can lead to more serious voltage sags that require placing more monitors. In addition, the optimal allocation schemes of these situations are different.

\section{Conclusion}

To solve the optimal allocation problem of PQMs in a power system, the voltage sag caused by different types of short-circuit faults is considered and the calculation method for fault voltage when the fault points are on the buses and lines is analyzed. An IAGA is presented to solve the minimum number of PQMs and to select the best positions. The allocation scheme that satisfies the observability of the entire network and the minimum number of monitors obtained by the proposed algorithm exhibits the character of diversity. The proposed IAGA can provide power system planners with several selections and can obtain the optimal scheme according to the redundancy evaluation method proposed in this study. The algorithm is verified on the IEEE30 bus system to prove its feasibility and effectiveness.

\section{References}

[1] H. G. Yang, X. Y. Xiao, J. Y. Liu. Issues and technology assessment on power quality Part 1: General concepts on power quality, J . Electric Power Automation Equipment.23(2003)1-4.

[2] S. Tao, X. N. Xiao, X. J. Liu. Study on distribution reliability considering voltage sags and scceptable indices, J. Proceedings of the CSEE.25(2005) 63-69.

[3] B. Liu, Q. Z. LI .Optimum Allocation of the Power Quality Monitors, J. Proceedings of the CSU-EPSA.21(2009) 69-73.

[4] O. Gabriel, V. Ferruccio, H. J. B. Math. An optimal monitoring program for obtaining voltage sag system indexes, J. .IEEE Transactions on Power Systems.21(2006)378-384.

[5] A. E. Mohamed, F. El-S. Ehab, M. A. S. Magdy, et al. A novel power quality monitoring allocation algorithm, J. IEEE Transactions Power Delivery.21(2006)768-777.

[6] W. Lu, L. J. Tian. Optimal allocation of voltage sag monitoring based on exposed area analysis ,J. Electric Power Automation Equipment.32(2012)45-50.

[7] A. A. Ibrahim, A. Mohamed, H. Shareef, et al. A new approach for optimal power quality monitor placement in power system considering system topology, J. Przeglad Elektrotechniczny.88(2012)272-276.

[8] D. J. Won, S. J. Ahn, I. Y. Chung, et al. A new algorithm to find the optimal numbers and locations of power quality monitors, C. Power Tech, St. Petersburg, Russia. IEEE. (2005) 1-7.

[9] Z. N. Wei, S. Wu, G. Q. Sun, et al. Optimal placement of power quality monitors based on multi-objective evolutionary algorithm, J. Power System Technology .36(2012),176-181.

[10] C. F. M. Almeida, N. Kagan. Allocation of power quality monitors by genetic algorithms and 
fuzzy sets theory, C. $15^{\text {th }}$ International Conference on Intelligent System Applications to Power Systems, Curitiba, Brazil: IEEE.(2009)1-6.

[11] M. Avendano-mora, J. V. Milanovic. Monitor placement for reliable estimation of voltage sags in power network, J. IEEE Transactions on Power Delivery.27(2012)936-944.

[12] Y. Liao. Fault location for single-circuit line based on bus-impedance matrix utilizing voltage measurements, J. IEEE Transactions on Power Delivery.23(2008)609-617.

[13] Y. Z. He, Z. Y. Wen. Power System Analysis (Volume 1).Wuhan : Huazhong University of Science and Technology Press,2002.

[14] X. F. Chang, L. J. Tian, Y. L. Qin. Method to accurately determine vulnerable areas of voltage sag, J. Electric Power Automation Equipment.31(2011)64-68.

[15] E. J. Elisa, H. Araceli, O. Gabriel. An approach based on analytical expressions for optimal location of voltage sags monitors, J. IEEE Transactions on Power Delivery.24(2009)2034-2042.

[16] L. Wang. Intelligent optimization algorithm and its application. Beijing : Tsinghua University Press,2001.

[17] Z. M. Sha, Y. Q. Hao, Y. S. Hao, et al. A new algorithm for PMU placement optimization in power system [J].Relay,2005,33(7):31-37.

[18] HE Lin, WANG Kejun, LI Guobin, et al. Elitist preserved genetic algorithm and its convergence analysis [J]. Control and decision,2000,15(1):63-66.

[19] Information on http://www.ee.washington.edu/research/pstca/, University of Washington.

WANG Hong, male, was born in Hunan Province in 1963. His main research interests include power quality and control, computer numerical control (CNC) technology, and information of equipment.

DAI Yu-xing, male, was born in Hunan Province in 1956. His main research interests include power quality and control, CNC technology, and information of equipment.

E-mail:whdy63@hotmail.com 